\title{
The Effect of Scalp Acupuncture on Sleeping Disorders in Autism
}

\author{
Chuen Heung Yau ${ }^{1 *}$, Cheuk Long Ip ${ }^{2}$, Yuk Yin Chau ${ }^{2}$ and Hong Zhang ${ }^{1}$ \\ ${ }^{1}$ Hu Nan University of Traditional Chinese Medicine. MD, BM, Lecturer, School of Chinese Medicine, Hong Kong University, Hong Kong \\ ${ }^{2}$ School of Chinese Medicine, Hong Kong Baptist University, Hong Kong
}

Submission: June 23, 2020 Published: July 21, 2020

*Corresponding author: Hong Zhang. Hu Nan University of Traditional Chinese Medicine. MD, BM, Lecturer, School of Chinese Medicine, Hong Kong University, Hong Kong

\begin{abstract}
Sleeping problems in children with Autism Spectrum Disorder (ASD) has been a concern as poor sleep may humper their daytime performance and even worsen the presentation of symptoms. Since acupuncture has been effective in treating insomnia in adults, we would like to know if children with ASD may also benefit better sleep quality from scalp acupuncture treatment. Our results showed that children with ASD generally obtain better sleep after regular scalp acupuncture therapy for 6 months. Participants have made significant improvements in bedtime resistance, sleep anxiety and daytime sleepiness domains.
\end{abstract}

Abbreviations: ASD: Autism Spectrum Disorders; ASMT: N-acetylserotonin O-methyltransferase; CSHQ: Children Sleeping Habit Questionnaire; GABA: $\gamma$-Aminobutyric Acid; SCN: Suprachiasmatic Nuclei; TCM: Traditional Chinese Medicine

\section{Introduction}

Autism Spectrum Disorder (ASD) is known as a pervasive developmental disorder. Young patients with ASD suffer impairments in social interaction, emotion control, communication skills, as well as restricted and repetitive behaviors. Despite sleep problems are not recognized as diagnostic criteria for ASD, many people reckon autistic children having difficulties in falling asleep, prolonged and repeated awakenings. According to a research by Krakowiak et al. [1], more than $50 \%$ of autistic children have sleep problem of bedtime resistance, insomnia and parasomnia. Sleep problems in children with ASD is prominent, in contrast to only $30 \%$ of typically developing children suffer sleep-related problems [2].

Circadian rhythm is disrupted in autistic children. Delayed sleep phase and irregular sleep-wake patterns are detected on young patients [3]. Since suprachiasmatic nuclei (SCN) in hypothalamus serve as biological clock, responsible for regulating daily and seasonal physiological rhythms. Alteration in function of SCN may cause disturbed cycle of sleepiness and alertness in ASD patients. Besides, neurohormone melatonin secreted by pineal gland also regulates circadian rhythm. Research detect abnormal melatonin level among autistic children $[4,5]$. An average diminished level of melatonin is measured in both daytime and night-time [6]. This deficit of melatonin production in ASD has been further reasoned to gene mutation of $\mathrm{N}$-acetylserotonin 0-methyltransferase (ASMT) gene [7].

In Traditional Chinese Medicine (TCM), acupuncture has long been used to treat poor sleep quality and insomnia in adults. Acupuncture refer to inserting needle onto specific locations of the body, i.e. acupoints, to achieve therapeutic effects. Systemic reviews and clinical reports have supported the use of the acupuncture on insomnia [8]. The mechanism of its action remains unclear. Neurotransmitters such as melatonin [9], endorphins [10], $\gamma$-aminobutyric acid (GABA) [11] and nitric oxide [12,13] are proved to response to acupuncture. Other than alteration in biochemical levels, autonomic nervous systems are also found to be directly modulated by acupuncture [14].

In a traditional acupuncture treatment for sleep problems, acupoints used are located both on the body and on the scalp. In recent decades, there have been theories developed supporting the sole use of acupoints on the scalp for treating brain-related diseases. Among all indications for scalp acupuncture therapy, both insomnia and ASD show prominent efficacy. In fact, in China 
and Hong Kong, acupuncture has been considered one of the common remedies for treating ASD. However, no investigation has been done on how scalp acupuncture therapy helps with the sleep problems in autistic children. This study therefore would like to know how scalp acupuncture influence the sleep problems frequently encountered by ASD patients.

\section{Subjects and Methods}

\section{Participants}

Participants are recruited from the patients consulted for Traditional Medicine treatment for autism at Hong Kong Baptist University Mr. \& Mrs. Chan Hon Yin Chinese Medicine Specialty Clinic and Good Clinical Practice Centre. Participants' age ranges from 2 to 11 years old. The participants must acquire a current medical diagnosis of autism issued by recognized specialists, i.e. psychiatrist or pediatrician.

\section{Therapist and treatment}

This study involves scalp acupuncture treatments and clinical assessments. The assessments and scalp acupuncture therapy sessions are carried out by principal investigator Ms. Yau Chuen Heung. She is an experienced Chinese medicine practitioner performing scalp acupuncture for autistic kids for more than eighteen years in Hong Kong.

During the procedure of scalp acupuncture therapy, young patients are placed on a chair or positioned in the bosom of their parents. The patients' heads are sterilized before inserting acupuncture needles $(0.20 \times 25 \mathrm{~mm})$ to the designated locations on the scalps. Acupoints of BaiHui (GV20), SiShenChong (EX-NH3), midline of forehead, lateral line 2 of forehead, posterior lateral line of vertex, primary auditory cortex, and auditory speech area are selected for the treatment of ASD. The needles are inserted obliquely $10 \mathrm{~mm}$ into the subcutaneous tissue at the acupoints. Participants stay at the therapeutic area for an hour after the insertion of the needles. Therapist swirl the needle every 15 minutes for a moderate stimulation to the acupoints. When the therapy ends, needles are removed from the scalp and discarded. Participants are asked to receive the therapy twice a week, not less than 30 sessions after 6 months.

Table 1

\begin{tabular}{|c|c|c|c|c|c|c|c|}
\hline \multirow{2}{*}{ Items } & \multicolumn{2}{|c|}{ Pre-treatment } & \multicolumn{2}{c|}{ Post-treatment } & \multicolumn{2}{c|}{ Change } \\
\hline Total score & Mean & SD & Mean & SD & Mean & SD & P-value \\
\hline Bedtime resistance & 54.67 & 7.91 & 50.95 & 8.14 & 3.71 & 6.46 & 0.02 \\
\hline Sleep onset delay & 12.43 & 2.73 & 11.52 & 3.03 & 0.9 & 1.34 & 0.01 \\
\hline Sleep duration & 1.95 & 0.59 & 1.81 & 0.68 & 0.14 & 0.65 & 0.33 \\
\hline Sleep anxiety & 4.67 & 1.74 & 4.33 & 1.49 & 0.33 & 1.11 & 0.18 \\
\hline Sleep wakings & 7.48 & 1.83 & 6.81 & 2.02 & 0.67 & 1.24 & 0.02 \\
\hline Parasomnia & 4.52 & 1.4 & 4.67 & 1.62 & -0.14 & 1.28 & 0.61 \\
\hline Sleep disordered breathing & 10.67 & 1.93 & 10.24 & 1.64 & 0.43 & 1.33 & 0.15 \\
\hline Daytime sleepiness & 1.33 & 1.49 & 4.05 & 1.28 & 0.29 & 0.96 & 0.19 \\
\hline
\end{tabular}

\section{Measurement of outcome}

Throughout the study, participants would attend two assessment sessions. In both sessions, a Children Sleeping Habit Questionnaire (CSHQ) is used to evaluate the sleep of the children with ASD. In the first session, parents are asked to complete CSHQ to assess the sleep condition of the child before the introduction of the treatment and 6 months prior. The second session is carried out 6 months later after receiving at least 30 sessions of acupuncture treatments.

CSHQ consists of 33 questions categorized into 8 domains, including bedtime resistance, sleep anxiety, sleep onset delay, sleep waking, sleep disordered breathing, parasomnia, daytime sleepiness and night waking. Parents are required to record the frequency of the respective items occurred in their children in the previous two weeks. A score will be marked according the frequency of the items [15].

Data analyses are conducted on all treatment responders. An alpha level of 0.05 was used for all statistical tests. The alteration in score of CSHQ throughout the studies were analyzed using paired t-test and ANOVA. All the calculations were performed on software IBM SPSS Statistics (Windows, version 21).

\section{Results}

The background score of the participants are analyzed by comparing the recalled performance 6 months prior to the study and at the beginning of the therapy. No significant alteration ( $p>0.05)$ in the sleep quality across all items is observed in the past 6 months before the start of the study.

The mean total score before and after treatment are higher than 41, which indicates unsatisfactory general sleep quality experienced by children with ASD [15]. We then compare the sleep before and after the introduction of scalp acupuncture treatment. Domains of bedtime resistance, sleep anxiety and daytime sleepiness have shown significant improvement $(\mathrm{p}<0.05)$ upon the treatment. No observable alternations have been made in items related to sleep onset delay, sleep duration, sleep wakings, parasomnia and sleep disordered breathing $(p>0.05)$ (Table 1$)$. 


\section{Discussion}

To date, no studies have investigated the effect of acupuncture on sleep of children with ASD. Our data demonstrated that scalp acupuncture is effective in alleviating or treating sleep-related problems in children with ASD. Participants are having generally better sleep quality after 6 months of therapy. They are observed to have improved bedtime resistance, sleep anxiety and daytime sleepiness. In fact, previous studies have noticed the tendency of late bedtime and early wake up time in autistic children $[1,16]$. Therefore, scalp acupuncture seems to have positive influence on those sleep problems common in autistic children. Children tends to become more tranquil at night-time and go to bed more cooperatively. At the same time, parents found their children less sleepy and have better attention during daytime activities.

Despite the mechanism of scalp acupuncture on the sleep pattern of children with ASD remains unclear, we suggest tends to relate sleep problems to emotion problems in autistic child. Items that have detected obvious improvement after treatment, i.e., bedtime resistance and sleep anxiety, are those aspects relevant to emotion and behavioral problems; Bedtime reluctance and sleep anxiety share common natures with aggressive behaviors and anxiety. On contrast, items less associated with emotions, such as parasomnia and sleep duration, show no noticeable alternation upon treatment. Besides, we also concern about nightmares reported by the children. We perceive dreams, especially horrible nightmares, are prominent implication of mental states and sleep quality. In reference to parents' reports, there is a drastic drop in frequency of nightmares after the acupuncture treatments. The above mentioned all cohere with the result obtained in our previous studies suggesting scalp acupuncture as an effective treatment in alleviating mood problems in young autistic patients. A better sleep might be the result of a better mood and behavior management upon receiving scalp acupuncture. We are convinced that the effect of scalp acupuncture on autistic children is general, not limited to specific defective aspects. This may explain numerous studies reporting acupuncture to be beneficial to vast array of developmental aspects in autistic children.

There are reports on short-term changes in sleep patterns of autistic children upon the introduction of acupuncture treatment. Some observed adverse sleep pattern after acupuncture [17]; while some suggest improvement in sleep pattern upon the therapy [18]. To our observation during this study, it is not rare for individuals to show changes in sleep pattern at the beginning of the scalp acupuncture therapy. Their response varies upon individuals at the first few treatment sessions. Some might show drowsy right after the sessions, while some become more arousal at night. These alteration in sleep pattern is brief and the changes diminish when the patient continue their routine treatment. This study shows that despite there might be variation in the transient effect of scalp acupuncture on children with ASD, patients may benefit a better sleeping pattern in the long run with a regular scalp acupuncture treatment.
There are few limitations in this study. Firstly, measurement of sleep quality is subjective to bias when using parent-reported questionnaires. Apart from questionnaires, sleep diary can also be used to record the detailed sleeping habits of the children. Instruments such as polysomnography and actigraphy should be utilized for an objective evaluation of sleep quality. Secondly, it would be preferable if there is a greater sample size. Since sleep condition varies among individuals, it would minimize the influence of individual variation.

\section{Conclusion}

This is the study that focuses on the therapeutic value of scalp acupuncture on sleep of ASD patients. It is recognized that difficulties encountered by autistic children are not limited to the typical presentations. They may experience deficits in various aspects crucial to live a normal daily life. We expect to see more efforts in developing novel and effective treating methods for holistic well-being of the autistic patients in the future.

\section{References}

1. Krakowiak P, Goodlin-Jones B, Hertz-Picciotto I, Croen LA, Hansen RL (2008) Sleep problems in children with autism spectrum disorders, developmental delays, and typical development: A population-based study. J Sleep Res 17(2): 197-206.

2. Richdale AL (1999) Sleep problems in autism: prevalence, cause, and intervention. Dev Med Child Neurol 41(1): 60-66.

3. Wiggs L, Stores G (2004) Sleep patterns and sleep disorders in children with autistic spectrum disorders: insights using parent report and actigraphy. Dev Med Child Neurol 46(6): 372-380.

4. Mulder EJ, Anderson GM, Kemperman RF, Oosterloo-Duinkerken A, Minderaa RB, et al. (2010) Urinary excretion of 5-hydroxyindoleacetic acid, serotonin and 6-sulphatoxymelatonin in normoserotonemic and hyperserotonemic autistic individuals. Neuropsychobiology 61(1): 2732.

5. Tordjman S, Anderson GM, Bellissant E, Botbol M, Charbuy H, et al. (2012) Day and nighttime excretion of 6-sulphatoxymelatonin in adolescents and young adults with autistic disorder. Psychoneuroendocrinology 37(12): 1990-1997.

6. Tordjman S, Najjar I, Bellissant E, Anderson G, Barburoth M, et al. (2013) Advances in the research of melatonin in autism spectrum disorders: literature review and new perspectives. Int J Mol Sci 14(10): 20508-20542.

7. Melke J, Botros HG, Chaste P, Betancur C, Nygren G, et al. (2008) Abnormal melatonin synthesis in autism spectrum disorders. Mol Psychiatry 13(1): 90.

8. Shergis JL, Ni X, Jackson ML, Zhang AL, Guo X, et al. (2016) A systematic review of acupuncture for sleep quality in people with insomnia. Complement Ther Med 26: 11-20.

9. Spence DW, Kayumov L, Chen A, Lowe A, Jain U, et al. (2004) Acupuncture increases nocturnal melatonin secretion and reduces insomnia and anxiety: a preliminary report. J Neuropsychiatry Clin Neurosci 16(1): 19-28.

10. Cheng CH, Yi PL, Lin JG, Chang FC (2011) Endogenous opiates in the nucleus tractus solitarius mediate electroacupuncture-induced sleep activities in rats. Evid Based Complement Alternat Med 2011: 159209.

11. Fu LW, Longhurst JC (2009) Electroacupuncture modulates vlPAG release of GABA through presynaptic cannabinoid CB1 receptors. J Appl Physiol 106(6): 1800-1809. 
12. Gao XY, Ma QL, Hu B (2007) Effects of acupuncture at" Sishencong"(EX-HN 1) on physiological functions in the sleep disorder model mouse. Zhongguo Zhen Jiu 27(9): 681-683.

13. Li S, Chen K, Wu Y, Jiao J, Tao L (2003) Effects of warm needling at zusanli (ST 36) on NO and IL-2 levels in the middle-aged and old people. J Tradit Chin Med 23(2): 127-128.

14. Huang W, Kutner N, Bliwise DL (2011) Autonomic activation in insomnia: the case for acupuncture. J Clin Sleep Med 7(1): 95-102.

15. Owens JA, Spirito A, McGuinn M (2000) The Children's Sleep Habits Questionnaire (CSHQ): psychometric properties of a survey instrument for school-aged children. Sleep 23(8): 1043-1052.
16. Allik H, Larsson JO, Smedje H (2006) Sleep patterns of school-age children with Asperger syndrome or high-functioning autism. J Autism Dev Disord 36(5): 585-595

17. Lee MS, Choi TY, Shin BC, Ernst E (2012) Acupuncture for children with autism spectrum disorders: a systematic review of randomized clinical trials. J Autism Dev Disord 42(8): 1671-1683.

18. Wong VC, Chen WX, Liu WL (2010) Randomized controlled trial of electro-acupuncture for autism spectrum disorder. Altern Med Rev 15(2): 136-146.
Your next submission with Juniper Publishers will reach you the below assets

- Quality Editorial service

- Swift Peer Review

- Reprints availability

- E-prints Service

- Manuscript Podcast for convenient understanding

- Global attainment for your research

- Manuscript accessibility in different formats

( Pdf, E-pub, Full Text, Audio)

- Unceasing customer service

Track the below URL for one-step submission https://juniperpublishers.com/online-submission.php 